INPLASY

PROTOCOL

To cite: Chao et al. Effect of Tai Chi on blood lipid in patients with cardiovascular disease: A systematic review and metaanalysis. Inplasy protocol 202230024. doi:

10.37766/inplasy2022.3.0024

Received: 06 March 2022

Published: 06 March 2022

Corresponding author: Mengyao Chao

2017020251@stu.sdnu.edu.cn

Author Affiliation:

Universiti Putra Malaysia.

Support: Chao Mengyao.

Review Stage at time of this submission: Data extraction.

Conflicts of interest:

None declared.

\section{Effect of Tai Chi on blood lipid in patients with cardiovascular disease: A systematic review and meta- analysis}

Chao, MY1; Xiang, CQ2 ; Ding, $\mathrm{M}^{3}$.

Review question / Objective: Effect of Tai Chi on blood lipids in patients with cardiovascular diseases.

Condition being studied: Dyslipidaemia significantly increases the risk of ischaemic cardiovascular morbidity, with approximately $10 \%$ of morbidity in the population attributable to high TC. previous studies have shown that Tai Chi has a positive effect on TC, HDL and LDL, and although there are only small effect sizes, they may all indicate clinically meaningful improvements. However, Tai Chi was only better in increasing HDL compared to aerobic exercise. Studies now suggest that conventional treatment + traditional Chinese exercise therapy (Tai Chi and Baduanjin) is smoothly in the middle of the range in terms of improving glycaemia and lipids, but the application of the results should be further validated clinically due to the risk of heterogeneity and bias.

INPLASY registration number: This protocol was registered with the International Platform of Registered Systematic Review and Meta-Analysis Protocols (INPLASY) on 06 March 2022 and was last updated on 06 March 2022 (registration number INPLASY202230024).

\section{INTRODUCTION}

Review question / Objective: Effect of Tai Chi on blood lipids in patients with cardiovascular diseases.

Rationale: Outbreaks of coronavirus disease 2019 (COVID-19) pose a greater threat to patients with cardiovascular disease, who are at high risk of developing severe disease. 2019 Outbreaks of coronavirus disease (COVID-19) pose a greater threat to patients with cardiovascular disease, who are at high risk of developing severe disease. A few systematic reviews have reported on the effects of tai chi on lipid markers, but most of the assessments are contradictory and 
more studies are needed to verify its effectiveness.

Condition being studied: Dyslipidaemia significantly increases the risk of ischaemic cardiovascular morbidity, with approximately $10 \%$ of morbidity in the population attributable to high TC. previous studies have shown that Tai $\mathrm{Chi}$ has a positive effect on TC, HDL and LDL, and although there are only small effect sizes, they may all indicate clinically meaningful improvements. However, Tai Chi was only better in increasing HDL compared to aerobic exercise. Studies now suggest that conventional treatment + traditional Chinese exercise therapy (Tai Chi and Baduanjin) is smoothly in the middle of the range in terms of improving glycaemia and lipids, but the application of the results should be further validated clinically due to the risk of heterogeneity and bias.

\section{METHODS}

Participant or population: People with cardiovascular diseases.

Intervention: A randomized controlled trial using Tai Chi as an intervention was conducted in the experimental group. The control group did not exercise or other aerobic exercise. Subjects were patients with cardiovascular disease. Ending indicators were at least one more lipid indicator.

Comparator: Comparing Tai Chi with no exercise. Comparing Tai Chi with Other exercise.

Study designs to be included: Randomized controlled trials.

Eligibility criteria: Inclusion criteria included the following: (1) randomised controlled trials with papers in English and Chinese; (2) studies on taiji interventions for cardiovascular disease; (3) taijiquan as the primary intervention in the experimental group and no exercise or a form of exercise other than taiji in the control group; (4) outcome indicators including at least one lipid indicator, and (5) articles published by
February 2022.Exclusion criteria included the following: (1) non-randomised controlled trials and observational trials; (2) exercise interventions other than tai chi in the experimental group; (3) papers with inconsistent or incomplete data for the main study indicators; (4) review papers, duplicate publications and papers where the full text could not be viewed; and (5) serious complications.

Information sources: Searches will be performed in the following 8 databases: PubMed/EMBASE/Cochrane Library/Web of Science/CNKI/VIP/WanFang Data/ MEDLINE. The search period will be from establishment to February 2022. All publications in English and Chinese will be included.

Main outcome(s): Total cholesterol (TC), triglycerides (TG), high-density lipoprotein (HDL), low-density lipoprotein(LDL).

Data management: Duration of intervention.

Quality assessment / Risk of bias analysis: The risk of bias of the included studies was assessed independently by two researchers (Chao and Xiang) using the Cochrane Collaboration System Evaluation Manual (version 5.1.0) criteria. The risk of bias scale included the following five entries: Random sequence generation (selection bias), Blinding of participants and personnel (performance bias), Blinding of outcome assessment (detection bias), Incomplete outcome data (attrition bias) and Selective reporting (reporting bias). Based on the information extracted from each eligible trial, each potential source of bias will be classified as high risk, unclear or low risk, and any disagreement will be determined by the third author (Ding).

Strategy of data synthesis: The Review Manage 5.4 software provided by Cochrane was applied to meta-analysis of the lipid data. As the outcome indicators were continuous variables, effect sizes were expressed using mean different (MD) and $95 \%$ confidence intervals $(\mathrm{Cl})$. The included studies were tested for heterogeneity using 
the X2 test. Homogeneity between studies was expressed as $\mathrm{P}>0.1,12<50 \%$ using a fixed effects model, while P50\% indicated significant heterogeneity and a random effects model was used. When heterogeneity between groups was too large, descriptive analyses were conducted.

Subgroup analysis: The subgroup analysis compares patients with different cardiovascular diseases to explore whether Tai Chi as an intervention has the same effect on patients with different cardiovascular diseases.

Sensitivity analysis: Sensitivity analysis was carried out using an exclusion-byexclusion method.

Language: Only Chinese and English literature is included.

Country(ies) involved: China, Malaysia.

Keywords: Tai Chi / Taiji Chuan / Cardiovascular disease / Blood Lipid / Systematic Review / Meta-analysis.

Contributions of each author:

Author 1 - Chao Mengyao.

Author 2 - Xiang, CQ.

Author 3 - Ding, $M$. 